\title{
Adiabatic melting of two-component Mott-insulator states
}

\author{
M. Rodríguez ${ }^{1,2}$, S. R. Clark ${ }^{1}$ and D. Jaksch ${ }^{1}$ \\ 1 Clarendon Laboratory, University of Oxford, Parks Road, Oxford OX1 3PU, U.K. and \\ ${ }^{2}$ ICFO-Institut de Ciències Fotòniques, 08860 Castelldefels (Barcelona), Spain
}

\begin{abstract}
We analyze the outcome of a Mott insulator to superfluid transition for a two-component Bose gas with two atoms per site in an optical lattice in the limit of slow ramping down the lattice potential. This manipulation of the initial Mott insulating state transforms local correlations between hyperfine states of atom pairs into multiparticle correlations extending over the whole system. We show how to create macroscopic twin Fock states in this way an that, in general, the obtained superfluid states are highly depleted even for initial ground Mott insulator states.
\end{abstract}

PACS numbers: 03.75.Lm, 03.75.Mn, 03.75.Dg

\section{INTRODUCTION}

The experimental realization of degenerate atomic gases [1] have opened up the possibility of engineering strongly correlated many-body quantum states. Such states are fundamental for the development of quantum technologies such as those implementing quantum information protocols and quantum computation. A paramount example is the realization of a Mott insulator (MI) 2, 3] in the lowest Bloch band of an optical lattice which can serve as a quantum register [4]. Bose-Einstein condensates of alkali atoms have also been proposed as systems to realize macroscopic superposition states [5] or number correlated states (also called twin Fock states) which are useful for quantum metrology $[6,7,6,8,9]$.

In [9] we proposed a method for engineering twin Fock states via a two-component MI to superfluid (SF) transition. The proposed scheme manipulates hyperfine states of atom pairs pinned to single lattice sites and decoupled from one another in the MI regime [4, 10]. The MI limit has already proven to be useful starting point for quantum state engineering since the motional dynamics are frozen out and the system decouples to a set of single site Hamiltonians to good approximation. In the case of a two-component system with doubly occupied sites spinchanging collisions will occur and can be accurately controlled with external magnetic or microwave fields [10]. As a result the spin-dynamics of atom pairs behaves like an effective two-level system undergoing Rabi-like oscillations. In contrast to the usual Rabi-model for the coupling of a pair of single-particle states this represents a coherent coupling between a pair of two-particle states induced by collisional interactions. These local two-particle correlations are then transformed into multi-particle correlations extending over the whole system by melting the MI into a SF. This quantum melting can be experimentally implemented by adiabatically ramping down the depth of the lattice potential.

The build up of long-range correlations when a periodic potential is dynamically lowered is a highly non trivial process that occurs rather quickly in a one-component system [2, 9, 11]. The quantum melting of a multicomponent system is still an open problem that is even more intriguing due to the different phases that appear in the $T=0$ phase diagram depending on the ratios of the interparticle interactions and of the tunneling rates of each component [12]. An analysis of the two-component symmetric case with all interparticle interactions equal can be found in [13]. In [9] we studied the adiabatic melting of an optical lattice in a two-component system with different values for the inter-species and intra-species interactions. We showed that one can create twin Fock states with high fidelity via melting of an initial MI state with two atoms per site in different internal states. We inspected possible sources of experimental imperfections such as atom loss during melting or depletion caused by final finite interactions. The possibility of creating macroscopic superposition states via the adiabatic melting of on-site entangled MI states in an infinitely connected lattice was also briefly considered in [9]. An examination of the adiabatic melting in an infinitely connected lattice, including the effect of the dynamical relative phase acquired by the state can be found in [14]. In this paper we extend the results in [9, 14] and analyze the melting of on-site superposition states in a lattice with only nearest neighbor hopping. We show that the final SF states obtained from such on-site superposition states are highly depleted even if the initial state is a ground MI state. For the melted states we calculate the momentum distribution and the momentum space particle number correlations. These quantities are experimentally accessible either by time of flight expansion of the cloud or via shot noise measurements [15].

This paper is organized as follows. In section [I] we introduce the two-mode Bose-Hubbard model (BHM) and the initial Mott insulating states that we consider. In section [II we describe the adiabatic approximation and the no-crossing rule that we use to infer the final superfluid states. In section IV we illustrate how one can create macroscopic twin Fock states if the inter-species interaction is greater than the intra-species interaction during melting. In $\mathrm{V}$ we show using the symmetry properties of the initial states and a series of approximations, that an on-site entangled MI ground state melts into a highly depleted SF state in the limit of intra-species interaction greater than the inter-species interaction. 


\section{TWO-COMPONENT BHM}

In this section we introduce the two-component BHM that describes the dynamics of ultra-cold atoms in an optical lattice. We discuss its experimental feasibility and analyze the symmetries of the Hamiltonian. Finally we introduce the initial MI states under consideration.

\section{A. Optical lattice setup}

Our focus is on a state-dependent optical lattice which is sufficiently deep that two hyperfine states $a$ and $b$ of the atoms are be trapped in the lowest Bloch band of the lattice. The resulting system is then accurately described 2] by the two-component BHM with the corresponding Hamiltonian $(\hbar=1)$

$$
\begin{gathered}
\hat{H}=-\sum_{\langle i, j\rangle}\left(J_{a} \hat{a}_{i}^{\dagger} \hat{a}_{j}+J_{b} \hat{b}_{i}^{\dagger} \hat{b}_{j}\right)+U \sum_{i} \hat{n}_{i}^{a} \hat{n}_{i}^{b} \\
+\frac{V_{a}}{2} \sum_{i} \hat{n}_{i}^{a}\left(\hat{n}_{i}^{a}-1\right)+\frac{V_{b}}{2} \sum_{i} \hat{n}_{i}^{b}\left(\hat{n}_{i}^{b}-1\right),
\end{gathered}
$$

where $\hat{a}_{i}\left(\hat{b}_{i}\right)$ is the bosonic destruction operator for an $a(b)$-atom localized in lattice site $i, \hat{n}_{i}^{a}=\hat{a}_{i}^{\dagger} \hat{a}_{i}$ and $\hat{n}_{i}^{b}=\hat{b}_{i}^{\dagger} \hat{b}_{i}$, while $\langle i, j\rangle$ denotes summation over nearestneighbors. The parameter $J_{a(b)}$ is the tunnelling matrix element for atoms in state $a(b) ; V_{a(b)}$ and $U$ are the on-site intra- and inter-species interaction matrix elements, respectively. A crucial feature of the optical lattice setup is that the ratio between the tunnelling and interaction matrix elements in Eq. (1) is experimentally controllable via the lattice laser intensities. Further independent control of the interaction matrix elements $U$, $V$ can be achieved with Feshbach resonances [16], or by shifting the $a$ and $b$ atoms away from each other using state-dependent lattices [4]. All of these methods of control can be exploited dynamically to permit different regimes of the Hamiltonian in Eq. (1) to be unitarily connected. Together with the long decoherence times of ultra-cold atoms this dynamical manipulation can often be implemented on near-adiabatic timescales.

Based on the considerable flexibility of the optical lattice setup we make a number of simplifications in our analysis. First, we restrict the couplings to the symmetric case where $J_{a}=J_{b}=J$ and $V_{a}=V_{b}=V$. Second, we consider states of the system $|\psi\rangle$ in which the lattice is commensurately filled with two atoms per site so $\hat{N}|\psi\rangle=N|\psi\rangle$ has $N=2 M$, where $\hat{N}=\sum_{i}\left(\hat{n}_{i}^{a}+\hat{n}_{i}^{b}\right)$ is the total particle number and $M$ is the total number of sites. Finally, we confine our attention to a 1D lattice with periodic boundary conditions (i.e. $M+1 \equiv 1$ ) in addition to the spatial homogeneity of the matrix elements in Eq. (11). This is not only a good approximation for the center of a large system, where trapping and boundary effects have a negligible influence, but is also directly applicable to recently demonstrated ring-shaped optical lattices [17].

\section{B. Symmetries}

The most basic conserved quantity of the twocomponent BHM is the total number of atoms $\hat{N}_{a}=$ $\sum_{i} \hat{n}_{i}^{a}, \hat{N}_{b}=\sum_{i} \hat{n}_{i}^{b}$ in the either of the internal states $a$ or $b$, respectively. With the additional assumptions outlined a number of other symmetries arise which we exploit in this work. The homogeneity and periodic boundary conditions assumed results in translational invariance. To exploit this we introduce quasi-momentum operators for each component as

$$
\begin{aligned}
& \hat{c}_{k}^{\dagger}=\frac{1}{\sqrt{M}} \sum_{i=1}^{M} \hat{a}_{i}^{\dagger} e^{-\mathrm{i} k r_{i}} \\
& \hat{d}_{k}^{\dagger}=\frac{1}{\sqrt{M}} \sum_{i=1}^{M} \hat{b}_{i}^{\dagger} e^{-\mathrm{i} k r_{i}}
\end{aligned}
$$

where $k$ is the quasi-momentum in the first Brillouin zone and $r_{i}$ is the position of the site $i$. The quasimomentum states spanning the first Brillouin zone have $k=\delta k \times\left\{-K_{m}, . ., 0, . ., K\right\}$ with $\delta k=2 \pi / M d$ and $d$ being the distance between lattice sites. For $M$ even $K_{m}=M / 2$ and $K=M / 2-1$, while for $M$ odd $K_{m}=K=(M-1) / 2$. The total momentum of the system is then $\hat{P}=\sum_{k} k\left(\hat{\mathrm{n}}_{k}^{a}+\hat{\mathrm{n}}_{k}^{b}\right)$, where $\hat{\mathrm{n}}_{k}^{a}=\hat{c}_{k}^{\dagger} \hat{c}_{k}$ and $\hat{\mathrm{n}}_{k}^{b}=\hat{d}_{k}^{\dagger} \hat{d}_{k}$, and is a conserved quantity. The total momentum generates cyclic shifts of the lattice sites, e.g. $i \mapsto i+1$ for the site indices of all operators, via the unitary $\hat{S}=\exp (-2 \mathrm{i} \hat{P} \pi / M)$. Its action on the bosonic creation operators is

$$
\begin{aligned}
& \hat{S} \hat{a}_{i}^{\dagger} \hat{S}^{\dagger}=\hat{a}_{i+1}^{\dagger}, \\
& \hat{S} \hat{b}_{i}^{\dagger} \hat{S}^{\dagger}=\hat{b}_{i+1}^{\dagger},
\end{aligned}
$$

simultaneously for all sites $i$. The homogeneity of the system also gives mirror reflection symmetry in which site indices for operators interchange as $i \leftrightarrow M-i+1$. In this case the parity operator $\hat{R}$ is the conserved quantity and implements the transformation

$$
\begin{aligned}
\hat{R} \hat{a}_{i}^{\dagger} \hat{R} & =\hat{a}_{M-i+1}^{\dagger}, \\
\hat{R} \hat{b}_{i}^{\dagger} \hat{R} & =\hat{b}_{M-i+1}^{\dagger},
\end{aligned}
$$

simultaneously for all sites $i$. Finally, the homogeneity and symmetry of the matrix elements result in color inversion symmetry $a \leftrightarrow b$. Like parity there is a hermitianunitary operator $\hat{C}$ which is the conserved quantity and implements the transformation

$$
\hat{C} \hat{a}_{i}^{\dagger} \hat{C}=\hat{b}_{i}^{\dagger}
$$

simultaneously for all sites $i$. These three symmetries are schematically represented in Fig. 1(a)-(c). 
(a)
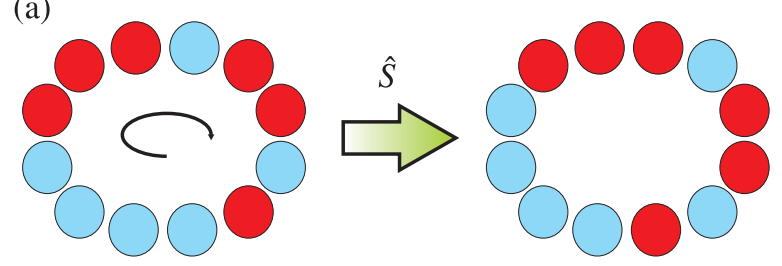

(b)

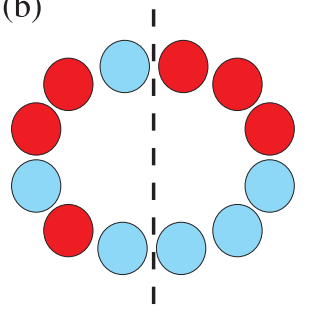

(c)

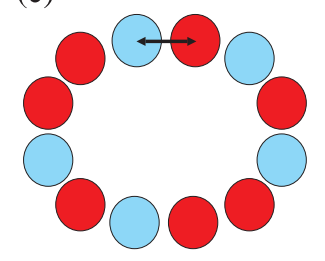

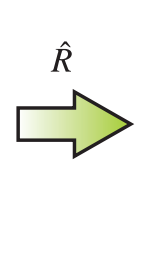
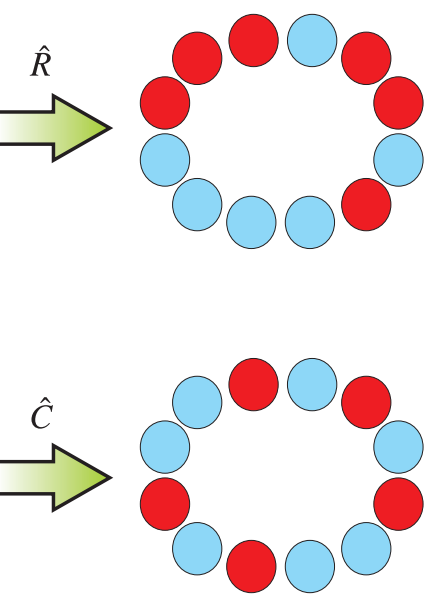

FIG. 1: Schematic representations of (a) cyclic shifting, (b) mirror reflection and (c) color inversion symmetry for a 1D lattice with periodic boundary conditions. The colors represent atoms in two different hyperfine states $a$ and $b$.

Symmetries are by definition related to the presence of degeneracies [18] or more precisely to a degeneracy which remains for all values of the couplings in the Hamiltonian - a so-called permanent degeneracy. By using these symmetries of $\hat{H}$ it follows that any of its eigenstates which are connected by a combination of these symmetry operators are necessarily degenerate. Moreover, any eigenstates which cannot be mapped on to each other by symmetry operators will be non-degenerate if the symmetries of the system are exhaustive [19]. Other types of degeneracies can occur when the couplings attain a particular value which indicates that at this point the system acquires a higher symmetry as compared to other points. This enhancement of symmetry frequently occurs in the limits of zero or infinite coupling. An important example in this work is when $J=0$ where $\hat{H}$ decouples into a set of single-site Hamiltonians and acquires full permutational symmetry. The breaking of the full permutational symmetry for finite $J$ splits the degenerate eigenstates of the $J=0$ Hamiltonian as we will see in proceeding sections.

Particle number conservation for each component breaks up the system Hilbert space into sectors where $\hat{N}_{a}|\psi\rangle=N_{a}|\psi\rangle$ and $\hat{N}_{b}|\psi\rangle=N_{b}|\psi\rangle$. The evolution of each component of an initial state in each of these sectors can therefore be treated independently. We shall use $\mathcal{H}_{n}$ to denote the sectors which possess the same to-

tal particle number $N$ where $N_{a}=N-n$ and $N_{b}=n$ using $n=0,1,2, \ldots, N$. We denote as $\mathcal{S}_{n}$ the completely symmetric subspace of the direct sum $\mathcal{H}_{n} \oplus \mathcal{H}_{N-n}$, defined by $\hat{S}|\psi\rangle=|\psi\rangle, \hat{R}|\psi\rangle=|\psi\rangle$ and $\hat{C}|\psi\rangle=|\psi\rangle$ for $|\psi\rangle \in \mathcal{H}_{n} \oplus \mathcal{H}_{N-n}$ where $n=0, \ldots, N / 2$. As we shall now outline for the specific initial states considered here we fix $N=2 M$ throughout and the only relevant sectors are those where $n$ is restricted to be even or $N / 2$.

\section{Initial states and MI ground states}

Our approach to exploit the two-component BHM to engineer quantum states is based on using two experimentally accessible initial states. Both of these states are generated in the MI limit where interactions are dominant, i.e. $U \gg J$ and $V \gg J$, and precisely with two atoms localized on every site. By controlling the spinchanging collisions a doubly occupied single-component MI formed in some additional hyperfine state can be transformed with near unit efficiency to the state [10]

$$
\left|\Psi_{a b}\right\rangle=\prod_{i=1}^{M}|a b\rangle_{i},
$$

with exactly the same number of $a$ and $b$ atoms in each lattice site. Note that $\left|\Psi_{a b}\right\rangle$ is exclusively contained in the symmetric subspace $\mathcal{S}_{M}$. By applying a further $\pi / 2$ Raman pulse to the state $\left|\Psi_{a b}\right\rangle$ an on-site entangled MI state

$$
\left|\Psi_{a a+b b}\right\rangle=\prod_{i=1}^{M} \frac{1}{\sqrt{2}}\left(|a a\rangle_{i}+|b b\rangle_{i}\right),
$$

can be created. This state is an equal superposition of all $2^{M}$ possible combinations of the atom- pair states $|a a\rangle$ and $|b b\rangle$ over $M$ sites. The states in the superposition are contained in the symmetric subspaces $\mathcal{S}_{2 n}$ with $n=0,1, \ldots,\lfloor M / 2\rfloor$, where $\lfloor\cdot\rfloor$ denotes the integer part. Specifically, in each sector $\mathcal{H}_{2 n}$ we form the state

$$
\left|\psi_{\mathrm{ps}}^{2 n}\right\rangle=\mathbb{P}\left(\prod_{i \leq n}|a a\rangle_{i} \prod_{n<j \leq M}|b b\rangle_{j}\right)
$$

where $\mathbb{P}(\cdot)$ stands for the normalized superposition of all permutations of the lattice sites. Since the state $\left|\psi_{\mathrm{ps}}^{2 n}\right\rangle$ possesses full permutational symmetry it is manifestly cyclic- and mirror-symmetric. If we then symmetrize the color as 33]

$$
\left|\Psi_{\mathrm{ps}}^{2 n}\right\rangle \equiv \frac{1}{\sqrt{2}}\left(\left|\psi_{\mathrm{ps}}^{2 n}\right\rangle+\left|\psi_{\mathrm{ps}}^{N-2 n}\right\rangle\right)
$$

we obtain states which are each contained in $\mathcal{S}_{2 n}$, respectively. The state $\left|\Psi_{a a+b b}\right\rangle$ can then be seen to be a binomial superposition of the states $\left|\Psi_{\mathrm{ps}}^{2 n}\right\rangle$ for all even- $n$ symmetric subspaces as

$$
\left|\Psi_{a a+b b}\right\rangle=\frac{1}{\sqrt{2^{M-1}}} \sum_{n=0}^{\lfloor M / 2\rfloor}\left(\begin{array}{c}
M \\
n
\end{array}\right)^{1 / 2}\left|\Psi_{\mathrm{ps}}^{2 n}\right\rangle .
$$


The two initial states in Eqs. (2) and (3) are exact symmetric ground states of the two-component BHM in the limit $J=0$ for different regimes of $U / V$. This is the MI regime where the particle-hole spectrum is gapped. In the case $U<V$ where intra-species interaction is dominant, and in the subspace $\mathcal{S}_{N / 2}$, the on-site state $|a b\rangle$ is energetically favored making $\left|\Psi_{a b}\right\rangle$ the nondegenerate ground state. This is easily confirmed by comparing it to all other configurations and moreover establishes that the ground state remains nondegenerate for non-zero $J$.

For the opposite case $U>V$ where the inter-species interaction is dominant "phase"-separated configurations composed of on-site states $|a a\rangle=|\circ\rangle$ and $|b b\rangle=|\bullet\rangle$ are energetically favored. Thus at $J=0$ the full permutational and color symmetry results in $2\left(\begin{array}{c}N / 2 \\ n\end{array}\right)$ degenerate ground states 34 spanned by all configurations of the $n$ states $|\circ\rangle$ and $N / 2-n$ states $|\bullet\rangle$ distributed amongst the $M=N / 2$ sites. Some of these states can be mapped by the cyclic shift symmetry $\hat{S}$, the mirror $\hat{R}$ or the color $\hat{C}$ transformation. We denote as $\mathcal{G}_{2 n} \subset \mathcal{S}_{2 n}$ the symmetrized (with respect to $\hat{C}, \hat{S}$ and $\hat{R}$ ) ground state manifold of the BHM in Eq. (1) for $J=0$ and $U>V$ with dimension denoted by $g(n, M)$.

On this basis we see that each of the states $\left|\Psi_{\mathrm{ps}}^{2 n}\right\rangle$ composing $\left|\Psi_{a a+b b}\right\rangle$ is one of many possible ground states in the symmetric subspace $\mathcal{G}_{2 n}$. However, as will be described in detail in Sec. $\mathrm{VA}$ once $J$ is non-zero this degeneracy is partially split resulting in $\left|\Psi_{a a+b b}\right\rangle$ mapping to a superposition of numerous low-lying excited states. This is schematically shown in fig. 2, The reason for this can be readily seen. For $0<J / U \ll 1$ nearestneighbor hopping will reduce the energy of configurations like $|\bullet \bullet \bullet \circ \circ \circ\rangle$ where $a$ and $b$ atoms form contiguous regions, compared to configurations like $|\bullet \circ \bullet \circ \bullet \circ\rangle$. Since $\left|\Psi_{a a+b b}\right\rangle$ contains contributions from all configurations it necessarily maps to a superposition of numerous lowlying excited states of the two-component BHM in Eq. (1) for each subspace $\mathcal{S}_{2 n}$ when $J$ is non-zero. This feature significantly complicates the analysis of this state even under adiabatic conditions.

\section{ADIABATIC QUANTUM MELTING}

Here we explore how adiabatic quantum melting which is an accessible coherent process that can be applied to the system and performs a highly non-trivial many-body unitary transformation [35] can be exploited as a means of quantum state engineering. For both initial states $\left|\Psi_{a b}\right\rangle$ and $\left|\Psi_{a a+b b}\right\rangle$ we take the quantum melting as ending in the non-interacting SF limit where $U=V=0$. In this limit the two-component BHM possesses a nondegenerate ground state

$$
\left|\Psi_{\mathrm{sf}}^{N_{a}, N_{b}}\right\rangle=\frac{\left(\hat{c}_{0}\right)^{N_{a}}\left(\hat{d}_{0}\right)^{N_{b}}}{\sqrt{N_{a} ! N_{b} !}}|\mathrm{vac}\rangle,
$$

for any sector $\mathcal{H}_{N_{b}}$ in which all the $a$ and $b$ atoms Bosecondense into their corresponding zero quasi-momentum

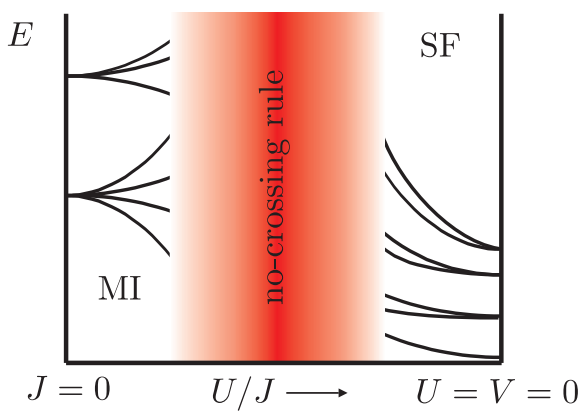

FIG. 2: Schematic energy-level structure for the twocomponent BHM with only one varying parameter $U / J$ and fixed $U / V$. The no-crossing rule assures that the system follows the instantaneous eigenstates and performs no level crossings if the states have all the same symmetry, there is only one varying parameter and the evolution is performed sufficiently slow.

state.

A feasible way to analyze the properties of the final melted states is to use the adiabatic approximation [20] in which the system follows the instantaneous eigenstates of the two-component BHM in Eq. (1) for each value $V / J$ and $U / J$ during the melting. The first requirement for the analysis to be tractable is the lack of level crossings. This condition is greatly aided by the no-crossing rule [19] which states that if the energy levels of a quantum system are plotted as a function of only one parameter, the curves for levels of the same symmetry do not cross. The no-crossing rule can be followed by using the flexibility of the optical lattice setup to ensure that the dynamical melting only varies the ratio $V / J$ whilst keeping $U / V$ constant. Thus the analysis can proceed by following the evolution of eigenstates in the symmetric subspace only. Also, the BHM is a non-integrable system that shows a chaotic spectrum and thus presents energy level repulsion 23]. The work in [23] provides numerical evidence that for small systems the symmetries we consider are exhaustive for the BHM (i.e. no crossings are seen). We extend this assumption to arbitrary sized systems. The energy levels may approach each other forming so-called avoided crossings, but for infinitely slow dynamics there will be no transitions between eigenstates. For finite times the Landau-Zener model gives the diabatic transition probabilities [21] between two levels, and this generalizes to Brundobler-Elser rule [22] for a multistate problem.

In principle, adiabatic evolution requires that the dynamical parameter $V / J$ changes in an infinitely long time. A crude estimate of the time scale $t_{r}$ needed for adiabatic melting of an optical lattice is given by $t_{r} \gg N V_{\max } / M J^{2}$ [1]], where $V_{\max } / J$ stands for the initial value in the MI regime. Importantly the adiabatic condition does not scale with the system size but with the density $N / M$. Numerical computations in 9] indicate that the adiabatic time scale is on the order of $t_{r} \sim 3 V_{\max } / J^{2}$ for a finite realistically sized one- 
dimensional system. These values are attainable in experimental setups and make the adiabatic approximation a reasonable assumption. These estimates are valid for the case when $U=0$ when Eq. (11) decouples as two onecomponent BHM's. Finding analogous time scales for the two-component system with $U \neq 0$ is still an open problem but it is reasonable to expect that the corresponding time scales are also attainable in experimental setups.

\section{MELTING THE PRODUCT MI : $\left|\Psi_{a b}\right\rangle$}

We consider first the melting of the product MI state $\left|\Psi_{a b}\right\rangle$. Our analysis begins by examining the outcome of quantum melting in a small system by using an exact numerical calculation. The results found then generalize to an arbitrarily large system and give rise to a final state whose usefulness for interferometry is analyzed.

\section{A. Exact numerics for a small system}

Here we provide some numerical evidence for the claims made in the previous section by performing an exact numerical diagonalization of the two-component BHM Hamiltonian $H$ for a system with $M=6$ sites. For the state $\left|\Psi_{a b}\right\rangle$ we need only consider the projection of $\hat{H}$ in the subspace $\mathcal{S}_{6}$. We then compute the ground state and low-lying excitations for a sequence of linearly decreasing interaction strengths $V / J$ with a fixed ratio $V / U=0.1$ throughout. In Fig. 3(a) the eigenenergies are shown confirming that the ground state in $\mathcal{S}_{6}$ remains nondegenerate during the melting, and that within the symmetric subspace level repulsion ensures that no level crossings occur consistent with the no-crossing rule. The final SF ground state is then $\left|\Psi_{\mathrm{sf}}^{6,6}\right\rangle$ in which the zero quasi-momentum mode of each component is equally occupied. Thus the quantum melting transforms local initial correlations between pairs of atoms into correlations between delocalized and macroscopically occupied modes. For this reason the final state $\left|\Psi_{\mathrm{sf}}^{6,6}\right\rangle$ is called a twin-Fock state [6]. In Fig. [3(b) the overlap $\left|\left\langle\psi_{\mathrm{gs}} \mid \Psi_{\mathrm{sf}}^{6,6}\right\rangle\right|$ between the instantaneous ground state $\left|\psi_{\mathrm{gs}}\right\rangle$ during the ramp and the SF twin-Fock $\left|\Psi_{\mathrm{sf}}^{6,6}\right\rangle$ is seen to converge to unity as a $V / J \rightarrow 0$.

\section{B. Large systems and interferometry}

We expand here the results that were investigated in [9]. By using the fact that $\left|\Psi_{a b}\right\rangle$ is a nondegenerate MI ground state and the no-crossing rule the outcome of adiabatic melting found for a small system can be readily generalized to any size system as

$$
\left|\Psi_{a b}\right\rangle \mapsto\left|\Psi_{\mathrm{sf}}^{N / 2, N / 2}\right\rangle=\left|\Psi_{\mathrm{tf}}\right\rangle,
$$

(a)

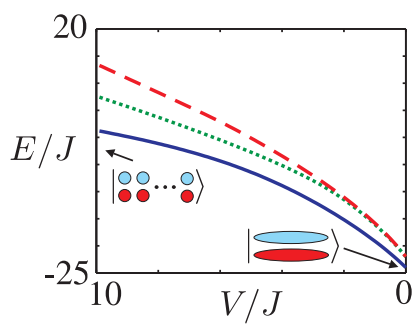

(b)

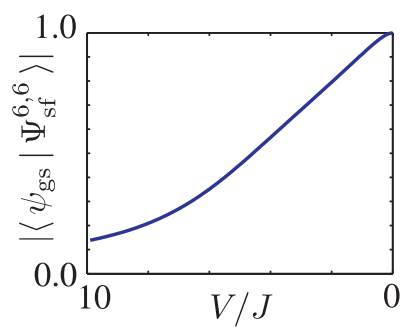

FIG. 3: (a) The spectrum of the two-component BHM $\hat{H}$ for $M=6$ sites in the symmetric subspace $\mathcal{S}_{6}$, i.e. with $N_{a}=N_{b}=6$, as a function of $V / J$ with $V / U=0.1$ throughout. To the far left (not plotted) where $V / J \gg 1$ the state $\left|\Psi_{a b}\right\rangle$ is the non-degenerate ground state. To the right where $V / J=0$ the non-interacting SF state $\left|\Psi_{\text {sf }}^{6}\right\rangle$ is the non-degenerate ground state. As expected these two ground states are adiabatically connected (solid line). The first and second excited states (dotted and dashed line, respectively) are also shown and become degenerate in the $V=0$ limit. (b) The overlap $\left|\left\langle\psi_{\mathrm{gs}} \mid \Psi_{\mathrm{sf}}^{6,6}\right\rangle\right|$ between the instantaneous ground state $\left|\psi_{\mathrm{gs}}\right\rangle$ and the SF state $\left|\Psi_{\mathrm{sf}}^{6,6}\right\rangle$ as a function of $V / J$.

(a)

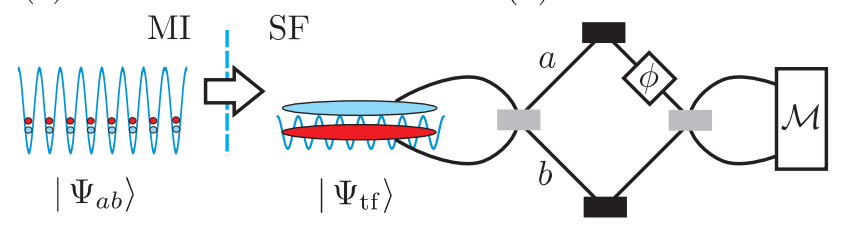

FIG. 4: (a) The melting of the product MI $\left|\Psi_{a b}\right\rangle$ in to the twin-Fock SF state $\left|\Psi_{\text {tf }}\right\rangle$. (b) The two internal states $a$ and $b$ represent the arms of an interferometer with the rotations R1, R2 and the phase-shift $\phi$ induced by appropriate laser pulses. Finally a $\phi$ dependent observable is measured at $\mathcal{M}$ from which $\phi$ can be deduced.

for arbitrary $N$. Quantum melting of the MI state $\left|\Psi_{a b}\right\rangle$ therefore provides a direct means of creating a $N$-particle twin-Fock state $\left|\Psi_{\mathrm{tf}}\right\rangle$, as depicted in Fig. 4(a).

Twin-Fock states [6] have been proposed as useful input states in interferometric experiments which could potentially allow measurement sensitivities which scale as $\propto 1 / N$. This is the Heisenberg limit for interferometry and improves on the standard limit obtained from an uncorrelated source where the sensitivity scales as $\propto 1 / \sqrt{N}$ [25, 26, 27]. A typical experimental scheme is a MachZenhder interferometer (MZI) that consists of an initial beam splitter and two interferometric paths that acquire a phase difference $\phi$ and are recombined in a final beam splitter, as shown in Fig. 4(b). The beam splitter operations could be implemented by rapid resonant $\pi / 2$ Raman pulses. Finally one extracts the phase difference measuring a phase dependent quantity.

To analyze this two-component system within the context of interferometry we will use the Schwinger boson 
formalism for constructing SU(2) operators [24, 25]

$$
\begin{aligned}
& \hat{J}_{x}=\frac{1}{2} \sum_{i}\left(\hat{a}_{i}^{\dagger} \hat{b}_{i}+\hat{b}_{i}^{\dagger} \hat{a}_{i}\right), \\
& \hat{J}_{y}=\frac{1}{2 \mathrm{i}} \sum_{i}\left(\hat{a}_{i}^{\dagger} \hat{b}_{i}-\hat{a}_{i}^{\dagger} \hat{b}_{i}\right), \\
& \hat{J}_{z}=\frac{1}{2}\left(\hat{N}_{a}-\hat{N}_{b}\right),
\end{aligned}
$$

and we denote $\hat{\mathbf{J}}=\left(\hat{J}_{x}, \hat{J}_{y}, \hat{J}_{z}\right)$. In many cases $\hat{J}_{z}$ is the phase-dependent measurement made in the interferometer. However, for the twin-Fock state $\langle\hat{\mathbf{J}}\rangle=0$ so a different quantity is required. One possibility is the next order angular momentum $\hat{J}_{z}^{2}$. Note that inefficient detection of such quantities can reduce the sensitivities back to the standard limit [28] and indirect measurement schemes of $\hat{J}_{z}^{2}$ are needed [29].

If we use $\hat{J}_{z}^{2}$ as the phase-dependent observable the resulting sensitivity can be calculated using error propagation theory. This gives $\Delta \phi=\Delta \hat{J}_{z}^{2} /\left|\partial\left\langle\hat{J}_{z}^{2}\right\rangle_{\phi} / \partial \phi\right|$ where $\left\langle\hat{J}_{z}^{2}\right\rangle_{\phi}$ and $\Delta \hat{J}_{z}^{2}=\left(\left\langle\hat{J}_{z}^{4}\right\rangle_{\phi}-\left\langle\hat{J}_{z}^{2}\right\rangle_{\phi}\right)^{1 / 2}$ are the average and the spread respectively. To calculate $\langle\cdot\rangle_{\phi}$ one can either rotate the state or the operators [25]. Since the action of the MZI is equivalent to the unitary operation $\exp \left(-i \phi \hat{J}_{y}\right)$, applying it to the operators gives

$$
\begin{aligned}
\left\langle\hat{J}_{z}^{2}\right\rangle_{\phi}= & \left\langle\hat{J}_{z}^{2}\right\rangle \cos ^{2}(\phi)+\left\langle\hat{J}_{x}^{2}\right\rangle \sin ^{2}(\phi) \\
& -\sin (\phi) \cos (\phi)\left\langle\left\{\hat{J}_{z}, \hat{J}_{x}\right\}\right\rangle,
\end{aligned}
$$

and

$$
\begin{aligned}
\left\langle\hat{J}_{z}^{4}\right\rangle_{\phi}= & \left\langle\hat{J}_{z}^{4}\right\rangle \cos ^{4}(\phi)+\left\langle\hat{J}_{x}^{4}\right\rangle \sin ^{4}(\phi) \\
& +\sin ^{2}(\phi) \cos ^{2}(\phi)\left\langle\left\{\hat{J}_{x} \hat{J}_{z}, \hat{J}_{z} \hat{J}_{x}\right\}\right. \\
& \left.+\left\{\hat{J}_{x}^{2}, \hat{J}_{z}^{2}\right\}+\left\{\hat{J}_{x}, \hat{J}_{z} \hat{J}_{x} \hat{J}_{z}\right\}\right\rangle \\
& -\cos ^{3}(\phi) \sin (\phi)\left\langle\left\{\hat{J}_{x}, \hat{J}_{z}^{3}\right\}+\left\{\hat{J}_{z}, \hat{J}_{z} \hat{J}_{x} \hat{J}_{z}\right\}\right\rangle \\
& -\sin ^{3}(\phi) \cos (\phi)\left\langle\left\{\hat{J}_{z}, \hat{J}_{x}^{3}\right\}+\left\{\hat{J}_{x}, \hat{J}_{x} \hat{J}_{z} \hat{J}_{x}\right\}\right\rangle,
\end{aligned}
$$

where $\{\hat{A}, \hat{B}\}=\hat{A} \hat{B}+\hat{B} \hat{A}$ is the anticommutator. For states which are zero eigenvectors of $\hat{J}_{z}$, such as twinFock states, this yields

$$
\Delta \phi^{2}=\frac{\sin ^{2} \phi\left(\left\langle\hat{J}_{x}^{4}\right\rangle-\left\langle\hat{J}_{x}^{2}\right\rangle^{2}\right)+\cos ^{2} \phi\left\langle\hat{J}_{x} \hat{J}_{z}^{2} \hat{J}_{x}\right\rangle}{4 \cos ^{2} \phi\left\langle\hat{J}_{x}^{2}\right\rangle^{2}} .
$$

The minimum value of this quantity occurs at $\phi=0$ and the sensitivity reduces to $\Delta \phi(0)=\frac{1}{2}\left\langle\hat{J}_{x}^{2}\right\rangle^{-1 / 2}$ after using the equality $\left\langle\hat{J}_{x} \hat{J}_{z}^{2} \hat{J}_{x}\right\rangle=\left\langle\hat{J}_{x}^{2}\right\rangle$ which holds for such states.

The sensitivity can be expressed entirely in terms of the one-particle density matrices $\rho_{i j}^{a}=\left\langle\hat{a}_{i}^{\dagger} \hat{a}_{j}\right\rangle$ and $\rho_{i j}^{b}=$ $\left\langle\hat{b}_{i}^{\dagger} \hat{b}_{j}\right\rangle$ using

$$
\left\langle\hat{J}_{x}^{2}\right\rangle=\frac{1}{4}\left[\sum_{i}\left(\rho_{i i}^{a}+\rho_{i i}^{b}\right)+\sum_{i, j}\left(\rho_{i j}^{a} \rho_{j i}^{b}+\text { h.c. }\right)\right] .
$$

For the initial (uncorrelated) MI state $\left|\Psi_{a b}\right\rangle$, which possesses no off-diagonal correlations $\rho_{i j}^{a}=\rho_{i j}^{b}=\delta_{i j}$, this yields a sensitivity at the standard limit $\Delta \phi=1 / \sqrt{2 N}$. For the final SF $\left|\Psi_{\mathrm{sf}}\right\rangle$, which possesses long-range correlations $\rho_{i j}^{a}=\rho_{i j}^{b}=1$, we see that asymptotically the Heisenberg limit $\Delta \phi=1 / \sqrt{N^{2} / 2+N}$ is recovered. Thus the scaling $\Delta \phi \propto N^{-\alpha}$ changes from $\alpha=1 / 2$ to $\alpha=1$ during the melting. An inspection of Eq. (8) shows that one needs non-zero values of the long-range correlations $\rho_{i j}$ in order to have better scaling than $N$ given by the first term in Eq. (8). We showed in [9] that one can obtain sensitivities approaching the HL scaling even in the presence of depletion if $U=0$. Recent results in 30] indicate that our conclusions about the effect of depletion are also valid for $U>0$. Moreover, we also showed in [9] that one can obtain HL sensitivities $(\alpha \sim 1)$ if particles are lost during the melting process.

\section{MELTING THE ENTANGLED MI : $\left|\Psi_{a a+b b}\right\rangle$}

In this section we analyze the melting of the entangled MI state $\left|\Psi_{a a+b b}\right\rangle$. In previous work [14] we demonstrated that this state may melt into a maximally entangled state if the lattice is infinitely connected. In a typical experimental set-up with only nearest neighbor hopping, the initial permutational symmetry for $J=0$ is broken for finite $J$ and the final SF state is highly excited. We begin by again considering the outcome of quantum melting in a small system. Finally after applying a number of approximations we compute the momentum correlations of the final melted state for a 1D lattice.

\section{A. Small system}

For $M=6$ the entangled MI state $\left|\Psi_{a a+b b}\right\rangle$ is an equal superposition of all 64 possible combinations of the atompair states $|\circ\rangle$ and $|\bullet\rangle$ over $M$ sites. Following Eq. (5) it can be written as

$$
\begin{aligned}
\left|\Psi_{a a+b b}\right\rangle= & \frac{1}{8}\left(\sqrt{2}\left|\Psi_{\mathrm{ps}}^{0}\right\rangle+\sqrt{12}\left|\Psi_{\mathrm{ps}}^{2}\right\rangle+\sqrt{30}\left|\Psi_{\mathrm{ps}}^{4}\right\rangle\right. \\
& \left.+\sqrt{20}\left|\Psi_{\mathrm{ps}}^{6}\right\rangle\right),
\end{aligned}
$$

where the phase-separated states in each subspace $\mathcal{S}_{2 n}$ are

$$
\begin{aligned}
\left|\Psi_{\mathrm{ps}}^{0}\right\rangle= & \mathbb{S}(|\bullet \bullet \bullet \bullet \bullet\rangle), \\
\left|\Psi_{\mathrm{ps}}^{2}\right\rangle= & \mathbb{S}(|\bullet \bullet \bullet \bullet \circ\rangle), \\
\left|\Psi_{\mathrm{ps}}^{4}\right\rangle= & \frac{1}{\sqrt{30}}[\sqrt{6} \mathbb{S}(|\bullet \bullet \circ \bullet \bullet\rangle)+\sqrt{12} \mathbb{S}(|\bullet \bullet \bullet \bullet \circ\rangle) \\
& +\sqrt{12} \mathbb{S}(|\bullet \bullet \bullet \bullet \circ\rangle)], \\
\left|\Psi_{\mathrm{ps}}^{6}\right\rangle= & \frac{1}{\sqrt{20}}[\sqrt{2} \mathbb{S}(|\bullet \circ \bullet \circ \bullet \circ\rangle)+\sqrt{12} \mathbb{S}(|\bullet \bullet \circ \bullet \circ\rangle) \\
& +\sqrt{6} \mathbb{S}(|\bullet \bullet \bullet \circ \circ \circ\rangle)] .
\end{aligned}
$$


(a)

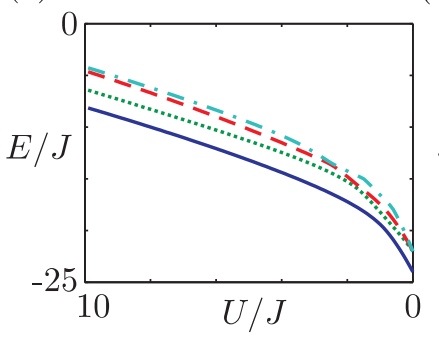

(b)

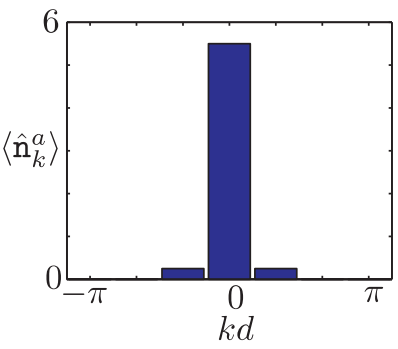

FIG. 5: (a) The low-energy spectrum of the two-component BHM $\hat{H}$ for $M=6$ sites in the symmetric subspace $\mathcal{S}_{4}$ as a function of $U / J$ with $V / U=0.1$ throughout. The initial state $\left|\Psi_{a a+b b}\right\rangle$ projected in this subspace is $\left|\Psi_{\mathrm{ps}}^{4}\right\rangle$. The adiabatic evolution of this state involves the ground state and two lowest-lying excited states. In the SF limit with $U=0$ the first-excited level is triply degenerate. Consistent with the no-crossing rule we see that energy levels within $\mathcal{S}_{4}$ repel each other and only avoided crossings are seen. (b) The momentum distribution $\left\langle\hat{\mathrm{n}}_{k}^{a}\right\rangle$ for one component (it does not matter which due to color symmetry) of the final adiabatically melted state $\left|\Psi_{\mathrm{f}}\right\rangle$ derived from $\left|\Psi_{a a+b b}\right\rangle$ with $M=6$.

Here we have used $\mathbb{S}(\cdot)$ to denote the normalized equal superposition of all equivalent configurations under the symmetry operations $\hat{S}, \hat{R}$ and $\hat{C}$. Each phase separated state $\left|\Psi_{\mathrm{ps}}^{2 n}\right\rangle$ is a member of $\mathcal{G}_{2 n}$ and is a superposition of all the $g$ inequivalent symmetric states within it (note that $g(0,6)=g(1,6)=1$, while $g(2,6)=g(3,6)=3$ ). The weights of this superposition Eq. (9) are given by the degeneracies due to symmetrization of these inequivalent states, which we denote by $s(l)$ where $l=1, ., g$.

We now proceed to melt each of these phase-separated contributions separately. For the subspaces $\mathcal{S}_{0}$ and $\mathcal{S}_{2}$ the corresponding states $\left|\Psi_{\mathrm{ps}}^{0}\right\rangle$ and $\left|\Psi_{\mathrm{ps}}^{2}\right\rangle$ are nondegenerate MI ground states. Analogous to the melting of $\left|\Psi_{a b}\right\rangle$ in the subspace $\mathcal{S}_{6}$ exact numerical diagonalization of the two-component BHM with $U>V$ confirms that there are no level crossings and so these two states are adiabatically connected to their corresponding symmetric SF ground states

$$
\begin{aligned}
& \mathbb{S}(|\bullet \bullet \bullet \bullet\rangle) \mapsto \frac{1}{\sqrt{2}}\left(\left|\Psi_{\mathrm{sf}}^{12,0}\right\rangle+\left|\Psi_{\mathrm{sf}}^{0,12}\right\rangle\right), \\
& \mathbb{S}(|\bullet \bullet \bullet \bullet\rangle) \mapsto \frac{1}{\sqrt{2}}\left(\left|\Psi_{\mathrm{sf}}^{10,2}\right\rangle+\left|\Psi_{\mathrm{sf}}^{2,10}\right\rangle\right) .
\end{aligned}
$$

In this notation $\mapsto$ we omit the dynamical phase $e^{i \alpha}$ acquired by the state during adiabatic melting [14]. In contrast subspaces $\mathcal{G}_{4}$ and $\mathcal{G}_{6}$ are three-fold degenerate and the states $\left|\Psi_{\mathrm{ps}}^{4}\right\rangle$ and $\left|\Psi_{\mathrm{ps}}^{6}\right\rangle$ are both composed of 3 inequivalent MI configurations. This situation becomes more typical with increasing system size. In the $J=0$ limit these configurations are degenerate, however once $J$ is non-zero they split in energy. In Fig. 5(a) the energies for the ground state and three lowest lying excitations in $\mathcal{S}_{4}$ are shown for a linear ramping of $U / J$ with $U / V=0.1$. This, together with a similar result for $\mathcal{S}_{6}$ (not shown), confirm that no level crossings occur. For this reason we can map the MI configurations via their ordering in energy with their corresponding ground state and excitations in the SF limit. The lowest energy configurations adiabatically connected to the symmetric SF ground state are

$$
\begin{aligned}
& \mathbb{S}(|\bullet \bullet \bullet \circ \circ\rangle) \mapsto \frac{1}{\sqrt{2}}\left(\left|\Psi_{\mathrm{sf}}^{8,4}\right\rangle+\left|\Psi_{\mathrm{sf}}^{4,8}\right\rangle\right), \\
& \mathbb{S}(|\bullet \bullet \bullet \circ \circ \circ\rangle) \mapsto\left|\Psi_{\mathrm{sf}}^{6,6}\right\rangle,
\end{aligned}
$$

respectively, while the two remaining configurations map to the lowest and second-lowest excitations in the SF limit. Quite generally there are many degenerate SF excitations in the $U=V=0$ limit. For the subspace $\mathcal{S}_{6}$ the first two lowest-lying excitations are degenerate, whereas the first three are for $\mathcal{S}_{4}$. To determine the appropriate state to which each configuration maps to we apply firstorder perturbation theory (see Appendix $\mathrm{A}$ ) in the interand intra-species interaction which splits these states and identifies the correct excitations. This then gives for $\mathcal{S}_{6}$

$$
\begin{aligned}
& \mathbb{S}(|\bullet \bullet \circ \bullet \circ \circ\rangle) \mapsto\left|\Psi_{\mathrm{ex}, 1}^{6}\right\rangle, \\
& \mathbb{S}(|\bullet \circ \bullet \circ \bullet \circ\rangle) \mapsto\left|\Psi_{\mathrm{ex}, 2}^{6}\right\rangle,
\end{aligned}
$$

and for $\mathcal{S}_{4}$ we have

$$
\begin{aligned}
& \mathbb{S}(|\bullet \bullet \bullet \bullet \circ\rangle) \mapsto\left|\Psi_{\mathrm{ex}, 1}^{4}\right\rangle \\
& \mathbb{S}(|\bullet \bullet \circ \bullet \bullet\rangle) \mapsto\left|\Psi_{\mathrm{ex}, 2}^{4}\right\rangle
\end{aligned}
$$

where $\left|\Psi_{\mathrm{ex}, 1}^{n}\right\rangle$ and $\left|\Psi_{\mathrm{ex}, 2}^{n}\right\rangle$ denote the lowest and secondlowest $\mathrm{SF}$ excitations in $\mathcal{S}_{n}$ (see Appendix $\mathrm{A}$ ). Note that this calculation of the lowest excitations works for arbitrary $M$, although it becomes cumbersome for larger system size as we would need a very large number of SF excitations.

Combining these results we can write the overall final SF state which is adiabatically connected to $\left|\Psi_{a a+b b}\right\rangle \mapsto$ $\left|\Psi_{\mathrm{f}}\right\rangle$ with $M=6$ as

$$
\begin{aligned}
\left|\Psi_{\mathrm{f}}\right\rangle= & \frac{1}{8}\left[e^{i \alpha_{0}}\left(\left|\Psi_{\mathrm{sf}}^{12,0}\right\rangle+\left|\Psi_{\mathrm{sf}}^{0,12}\right\rangle\right)+\sqrt{6} e^{i \alpha_{2}}\left(\left|\Psi_{\mathrm{sf}}^{10,2}\right\rangle+\left|\Psi_{\mathrm{sf}}^{2,10}\right\rangle\right)+\sqrt{6} e^{i \alpha_{4}}\left(\left|\Psi_{\mathrm{sf}}^{8,4}\right\rangle+\left|\Psi_{\mathrm{sf}}^{4,8}\right\rangle\right)+\sqrt{12} e^{i \alpha_{4,1}}\left|\Psi_{\mathrm{ex}, 1}^{4}\right\rangle\right. \\
& \left.++\sqrt{6} e^{i \alpha_{4,2}}\left|\Psi_{\mathrm{ex}, 2}^{4}\right\rangle+\sqrt{6} e^{i \alpha_{6}}\left|\Psi_{\mathrm{sf}}^{6,6}\right\rangle+\sqrt{12} e^{i \alpha_{6,1}}\left|\Psi_{\mathrm{ex}, 1}^{6}\right\rangle+\sqrt{2} e^{i \alpha_{6,2}}\left|\Psi_{\mathrm{ex}, 2}^{6}\right\rangle\right]
\end{aligned}
$$




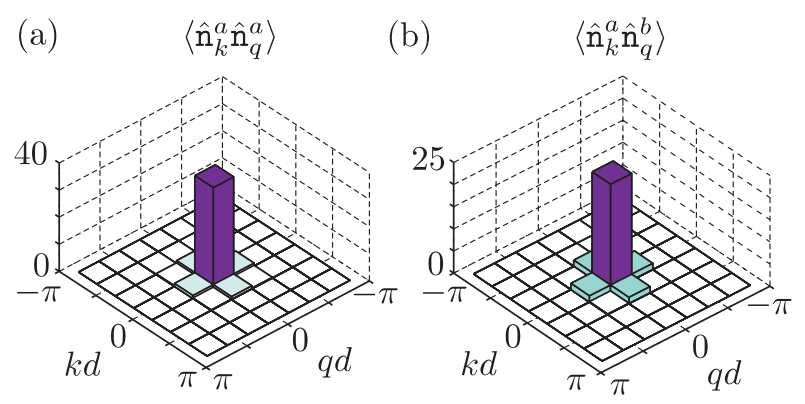

FIG. 6: (a) Momentum space particle number correlations $\left\langle\hat{\mathrm{n}}_{k}^{a} \hat{\mathrm{n}}_{q}^{a}\right\rangle$ of the final melted state $\left|\Psi_{\mathrm{f}}\right\rangle$ for one component (it does not matter which due to color symmetry) for $M=6$. (b) The inter-species momentum space particle number correlations $\left\langle\hat{\mathrm{n}}_{k}^{a} \hat{\mathrm{n}}_{q}^{b}\right\rangle$ for $\left|\Psi_{\mathrm{f}}\right\rangle$.

where we have now written explicitly the dynamical phases acquired by each of the states in the superposition. An observable of experimental significance is the momentum distribution for either component. In fact the momentum distribution $\left\langle\hat{\mathrm{n}}_{ \pm k}^{a}\right\rangle=\left\langle\hat{\mathrm{n}}_{ \pm k}^{b}\right\rangle$ is not dependent on the precise form of the excited states $\left|\Psi_{\text {ex }}\right\rangle$ since all states in the same degenerate manifold have the same expectation value $\left(\left\langle\hat{\mathrm{n}}_{ \pm \frac{\pi}{3 d}}^{a}\right\rangle=\frac{1}{2}\right.$ in this case). The resulting momentum distribution is shown in Fig. 5 (b), and gives the fraction of the population outside the zero-momentum mode (i.e. depletion of the Bose-condensate) for either component as $D=1 / 12 \approx 8.3 \%$. Both the intra-species, $\left\langle\hat{\mathrm{n}}_{ \pm k}^{a} \hat{\mathrm{n}}_{ \pm q}^{a}\right\rangle$, and the inter-species, $\left\langle\hat{\mathrm{n}}_{ \pm k}^{a} \hat{\mathrm{n}}_{ \pm q}^{b}\right\rangle$, momentum space particle number correlations do depend on the precise form of the excited states $\left|\Psi_{\text {ex }}\right\rangle$. Neither the momentum distributions nor the momentum space particle number correlations depend on the relative dynamical phases. In Fig. 6(a)(b) these correlations are plotted using the excited states determined from first-order perturbation theory with $U / J=0.01$ and $V / U=0.1$.

\section{B. Large systems}

The final SF state obtained via adiabatic melting the entangled MI state $\left|\Psi_{a a+b b}\right\rangle$ is given by

$$
\left|\Psi_{\mathrm{f}}\right\rangle=\frac{1}{\sqrt{2^{M-1}}} \sum_{n=0}^{\lfloor M / 2\rfloor}\left(\begin{array}{c}
M \\
n
\end{array}\right)^{1 / 2}\left|\Psi_{\mathrm{f}}^{2 n}\right\rangle,
$$

where $\left|\Psi_{\mathrm{ps}}^{2 n}\right\rangle \mapsto\left|\Psi_{\mathrm{f}}^{2 n}\right\rangle$ if we assume that $U / V$ is fixed during melting and that the symmetries considered are exhaustive and thus no level crossings occur. The numerical calculation for $M=6$ in section $\overline{V A}$ hints that it is very complicated to obtain the final state for larger $M$ which are inaccessible with exact numerics. Resolving the exact splitting of the states in each subspace $\mathcal{S}_{2 n}$ in the MI limit and in the SF limit is both complex and impractical for an arbitrarily large $M$. Instead, we introduce here some approximations that allow us to calculate the momentum distributions and momentum correlations of the final states.

In the MI regime for $J=0$ and $U>V$ the colorsymmetric ground state manifold is $\left(\begin{array}{c}M \\ n\end{array}\right)$ degenerate. For finite $J$, the degeneracy is broken and $g(n, M)$ states (obtained after symmetrization in $\hat{S}, \hat{R}$ ) are not coupled by the evolution. As shown below for large $M$ we can approximate the initial MI state $\left|\Psi_{\mathrm{ps}}^{2 n}\right\rangle$ as an equal superposition of the $g(n, M)$ states. In this way, using the adiabatic approximation we can map these $g$ states into the lowest $g$ states in the SF regime. We calculate the lowest eigenstates of the BHM in the SF regime $U=V=0$ and their degeneracies $f(l)$ such that $\sum_{l} f(l)=g$. Even without calculating the exact coefficients of the final $g$ states in the SF eigenbasis, we can compute the momentum distribution and the momentum space particle number correlations of the final melted state to good approximation.

\section{MI limit}

If $U / V>1$ and $J=0$ the phase-separated state $\left|\Psi_{\mathrm{ps}}^{2 n}\right\rangle$ belongs to the ground state manifold $\mathcal{G}_{2 n} \subset \mathcal{S}_{2 n}$. It is constructed as an equal superposition of the $\left(\begin{array}{c}N / 2 \\ n\end{array}\right)$ atompair states $|\circ\rangle$ and $|\bullet\rangle$ distributed over $M=N / 2$ sites. These atom-pair states belong to the ground state manifold. However, only $g(n, M) \leq\left(\begin{array}{c}N / 2 \\ n\end{array}\right)$ of them define the symmetrized ground-state manifold $\mathcal{G}_{2 n}$ and are not coupled by evolution for finite $J$. We showed in section VA that e.g. $g(3,6)=3$ and that for $M=6$, the state $\left|\Psi_{\mathrm{ps}}^{6}\right\rangle$ is a superposition of 3 states that evolve into 3 different final SF states. In general, we can calculate the dimension of the subspace $\mathcal{G}_{2 n}, g(n, M)$, by noticing that it is isomorphic to the number of color symmetric configurations in a bracelet (c.f. Fig.1) with $n$ beads of one color and $M-n$ beads of the other color. Such mathematical structures have been extensively studied [31, 32] and there are analytical formulas available for their properties. For even $M$, the number of complementable bracelets with $M$ beads and half of them of each color is given by [31]

$$
\begin{aligned}
& g(M / 2, M)=\frac{1}{2}\left[F\left(\frac{M}{2}\right)+2^{\frac{M}{2}-2}+\left(\begin{array}{c}
\frac{M}{2}-1 \\
\frac{M-2}{4}
\end{array}\right)\right] \frac{M}{2} \text { odd } \\
& g(M / 2, M)=\frac{1}{2}\left[F\left(\frac{M}{2}\right)+2^{\frac{M}{2}-2}+\left(\begin{array}{c}
\frac{M}{2} \\
\frac{M}{4}
\end{array}\right)\right] \frac{M}{2} \text { even, }
\end{aligned}
$$

where $F(n)=\frac{1}{2 n} \sum_{d \mid n} \Phi(n / d)\left(\begin{array}{c}2 d-1 \\ d-1\end{array}\right)+\Phi(2 n / d) 2^{d-1}$ and $\Phi(x)$ is Euler's totient function. For bracelets with odd total number of beads $M$ or even $M$ and $n \neq M / 2$, the number of complementable bracelets is given by [32]

$$
g(n, M)=\frac{1}{2}[\operatorname{Fold}(G, 0,\{d: d|M \cap d| n\})+H(n, M)] .
$$

Here, $\operatorname{Fold}\left(G(\cdot, \cdot), x,\left\{a_{1}, \ldots, a_{k}\right\}\right)$ gives the last term of the list $\left\{x, G\left(x, a_{1}\right), G\left(G\left(x, a_{1}\right), a_{2}\right), \ldots\right\}$ generated by the cumulative application of the function

$$
G(x, y)=x+\frac{\phi(y)}{M}\left(\begin{array}{c}
\frac{M}{y} \\
\frac{n}{y}
\end{array}\right)
$$



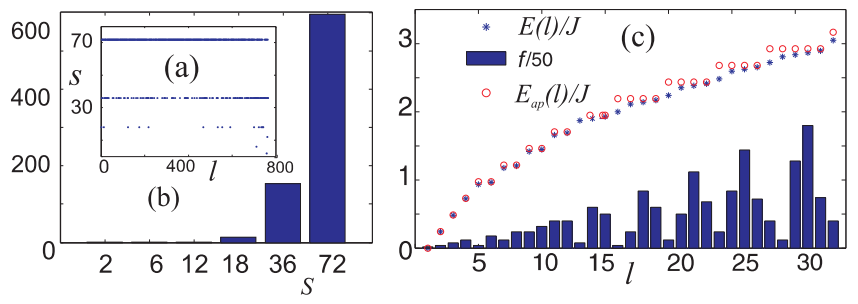

FIG. 7: (a) Number of MI ground states $\left(d_{M I}\right)$ that belong to each of the $g(9,18)=765$ independent states for finite $J$ for a lattice with $N / 2=N_{a}=N_{b}=18$ and $U>V$. (b) Number of independent states with a given degeneracy $d_{M I}$. The total number of independent states is equal to $g(9,18)=765$. (c) First energy levels $(*)$ in the SF regime $(U=V=0)$ and their degeneracy $f$ (bar plot) for $M=18$. The points (o) show the quadratic approximation to the dispersion relation. We plot only the first states such that $\sum_{l} f(l)=g$ for $g=765$.

to the elements of the input list $\left\{a_{1}, \ldots, a_{k}\right\}$ and

$$
H(n, M)= \begin{cases}\left(\frac{M-2}{2}\right) & \text { if } M \text { even, } n \text { odd } \\ \left(\frac{n-1}{2\rfloor}\right\rfloor & \text { otherwise. }\end{cases}
$$

Note that we recover $g(2,6)=g(3,6)=3$ obtained for the states $\left|\Psi_{\mathrm{ps}}^{4}\right\rangle$ and $\left|\Psi_{\mathrm{ps}}^{6}\right\rangle$ given in Sec VA.

\section{Superposition states}

We now need to write our initial state $\left|\Psi_{\mathrm{ps}}^{2 n}\right\rangle$ as a superposition of the $g(n, M)$ states that evolve independently for finite $J$. Using the no-crossing rule we can map the initial superposition $\left|\Psi_{\mathrm{ps}}^{2 n}\right\rangle$ at $J=0$ into a final superposition $\left|\Psi_{\mathrm{f}}^{2 n}\right\rangle$. The coefficients of the superposition are given by the the number of bracelet configurations $s(l)$ that can be obtained by mirror inversion $\hat{R}$ or cyclic permutation $\hat{S}$ of any of the independent states $l=1, . ., g(n, M)$ in the subspace $\mathcal{G}_{2 n}$. We show $s(l)$ for $M=18$ in Fig. 7 (a). Note that $\sum_{l}^{g} s(l)=\left(\begin{array}{c}M \\ n\end{array}\right)$. Numerical results in Fig. 7 (b) show that most (77\%) of the $g$ inequivalent configurations have the same degeneracy $s$ and that this behavior increases with size $M$ (for $M=22$ already $92 \%$ of the $g$ bracelets present the same degeneracy). We can thus assume that the number of states isomorphic to each independent configuration is constant $s=\left(\begin{array}{c}M \\ n\end{array}\right) / g$. Within this approximation, $\left|\Psi_{\mathrm{f}}^{2 n}\right\rangle$ is thus an equal superposition of the first $g$ eigenstates of the $\mathrm{SF}$ Hamiltonian.

\section{SF limit}

In Appendix B we show how to calculate the eigenstates $\left|\left\{e_{l}\right\}\right\rangle$ of the Hamiltonian (11) in the SF regime where $U=V=0$. The lowest eigenstates in ascending order in energy for large $M$ can be calculated efficiently (a)

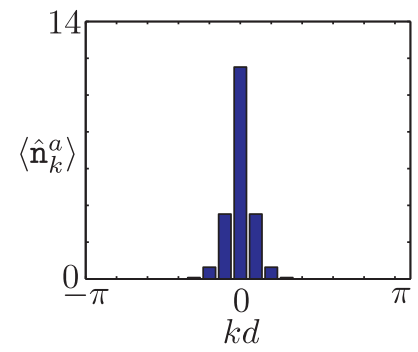

(b)

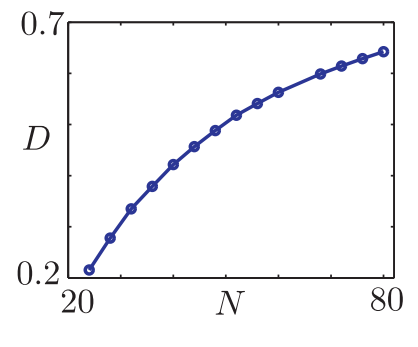

FIG. 8: (a) The momentum distribution of the final SF state $\left|\Psi_{\mathrm{f}}\right\rangle$ obtained via adiabatically melting the MI state $\left|\Psi_{\mathrm{aa}+\mathrm{bb}}\right\rangle$ with $N_{a}=N_{b}=M=20=N / 2$ and $U / V>1$. (b) The depletion $D=1-\left\langle\mathrm{n}_{0}^{a}\right\rangle / N_{a}$ of the melted state $\left|\Psi_{\mathrm{f}}^{N / 2}\right\rangle$ for $U / V>1$ as a function of the number of particles $N$. The solid line shows the best logarithmic fit $-0.0748(\ln N / 2)^{2}+$ $0.8201 \ln N / 2-1.3633$ to the calculated values (shown as o).

assuming a quadratic approximation $E_{a p}(l)$ to the noninteracting single-particle energy $E(l)$. Due to the color symmetry, the SF eigenstates $\left|e_{l}\right\rangle$ are degenerate. The degeneracies of the states $f(l)$ can be calculated numerically. We show in Fig. 7 (c) the eigenenergies of the first SF states and their degeneracies for $M=18$.

To obtain the momentum distribution of $\left|\Psi_{\mathrm{f}}^{2 n}\right\rangle$ we do not need to compute the exact distribution of the final

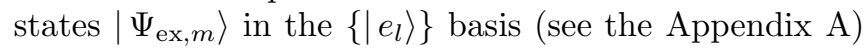
because all states in the same degenerate manifold have the same expectation value $\left\langle\hat{\mathrm{n}}_{q}^{a}\right\rangle=\left\langle\hat{\mathrm{n}}_{q}^{b}\right\rangle$. We just need to calculate the momentum distribution of the final eigenstates $\left\{\left|e_{l}\right\rangle\right\}$ and their degeneracies $f(l)$ and sum their contributions up to the highest excited state such that $\sum_{l} f(l) \approx g$.

On the other hand, we saw in Sec. $\nabla A$ that we need the exact form of the final states $\left|\Psi_{\text {ex }, m}\right\rangle$ in order to obtain the momentum-number correlations. Note, however, that $\sum_{m=1}^{f}\left\langle e_{m}\left|\hat{\mathrm{n}}_{k} \hat{\mathrm{n}}_{q}\right| e_{m}\right\rangle=$ $\sum_{m=1}^{f}\left\langle\Psi_{\mathrm{ex}, m}\left|\hat{\mathrm{n}}_{k} \hat{\mathrm{n}}_{q}\right| \Psi_{\mathrm{ex}, m}\right\rangle$ where we sum over a complete degenerate manifold. Thus, if we make the further approximation that the state $\left|\Psi_{\mathrm{f}}^{2 n}\right\rangle$ fully populates the highest excited manifold we can calculate its momentum space particle number correlations without knowing the exact states $\left|\Psi_{\mathrm{ex}, m}\right\rangle$. This is justified for large $g$ because the exact form of the highest excited states is concealed by the lower manifolds that are fully populated.

We show the momentum distribution of the melted state $\left|\Psi_{\mathrm{f}}\right\rangle$ in Fig. 8 (a). The final state is highly depleted. A plot of the depletion $D=1-\left\langle\mathrm{n}_{0}\right\rangle / N$ of the term $\left|\Psi_{\mathrm{f}}^{N / 2}\right\rangle$ as a function of $N$ is shown in Fig. 8 (b). It indicates the behavior of the depletion of the full state $\left|\Psi_{\mathrm{f}}\right\rangle$ because this term has the highest binomial coefficient in the sum (10) and a very large $g$. One can see that the depletion increases very rapidly with the number of particles.

The momentum-space particle number correlations of the melted state $\left|\Psi_{\mathrm{f}}\right\rangle$ are shown in figure 9. We observe 

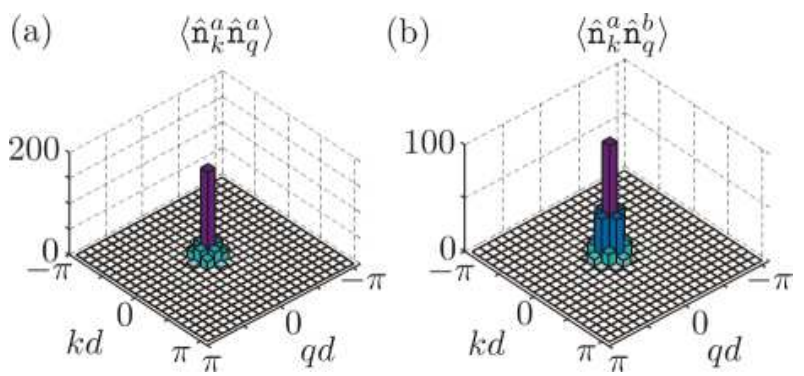

FIG. 9: (a) The momentum correlations $\left\langle\hat{\mathbf{n}}_{k}^{a} \hat{\mathbf{n}}_{q}^{a}\right\rangle$ for one mode for the state $\left|\Psi_{\mathrm{f}}\right\rangle$ obtained via the adiabatic melting of $\left|\Psi_{a a+b b}\right\rangle$ with $N=40$ and $U / V>1$. (b) The inter-species momentum correlations $\left\langle\hat{\mathrm{n}}_{k}^{a} \hat{\mathrm{n}}_{q}^{b}\right\rangle$ of $\left|\Psi_{\mathrm{f}}\right\rangle$ with $N=40$. Note that a twin-Fock state (7) would only show a central peak in both distributions.

that the population outside the zero momentum mode is higher for the inter-species momentum correlations. Our calculations clearly indicate that melting two component Mott insulators leads to quantum states with a complicated structure and long range correlations. A number of approximations were necessary to arrive at these results. We believe that an exact treatment is unlikely to reveal a simple structure which allows an intuitive interpretation of the melting process.

\section{SUMMARY}

We have analyzed the quantum melting of correlated two-component MI states into the SF regime using the adiabatic approximation. Based on the experimental fact that the one component MI-SF transition can be performed near adiabatically, we have applied it to the twomode case. Using the no-crossing rule we have shown that quantum melting of a two-component MI in an optical lattice provides a viable route for engineering twin Fock states useful in interferometric experiments. The twin Fock states present only macroscopic occupation of the final SF ground state although in general this is not the case. We have shown that due to the breaking of the translational symmetry when there is nearest neighbor hopping the final SF connected to a ground initial state can be highly depleted even in the limit of adiabatic behavior.

Using a set of well justified approximations, we have calculated the momentum correlations and momentum space particle number correlations for the final states obtained via melting on-site entangled states created in the MI regime. Our results clearly indicate that the melted SF states present a complicated structure and long range correlations. Further work is needed to ascertain if these correlations can be exploited for interferometry or other tasks.

\section{Acknowledgements}

M. R. thanks K. Surmacz, J.J. García-Ripoll and R. A. Molina for fruitful discussions and the Spanish MCyT under programme Juan de la Cierva for support. This research was supported by the EPSRC projects EP/E041612/1 and EP/C51933/1, and by the National Science Foundation under Grant No. NSF PHY0551164. M. R. and D. J acknowledge support from the EU through the STREP project OLAQUI.

\section{APPENDIX A: SPLITTING DEGENERATE SF EXCITATIONS}

In the non-interacting SF limit where $U=V=0$ the excited states are made up by expelling pairs of atoms of opposite momentum from the ground state Eq. (6). Of particular relevance to the exact calculation of the melting of $\left|\Psi_{a a+b b}\right\rangle$ in a small system are excitations in the subspaces $\mathcal{S}_{M}$, where $\left|N_{a}-N_{b}\right|=0$, and $\mathcal{S}_{M-2}$, where $\left|N_{a}-N_{b}\right|=4$ and $M=N / 2$. Here we shall demonstrate that the lowest-lying excitations in $\mathcal{S}_{M}$ and $\mathcal{S}_{M-2}$ are split by the inter- and intra-species interaction to firstorder in $V / J$ and $U / J$.

For the subspace $\mathcal{S}_{M}$ the two lowest-lying excitations are degenerate. These form an excitation subspace which is spanned by states where a single pair of atoms, of either component, is expelled from the condensate to equal and opposite momenta with the lowest possible magnitude as

$$
\begin{aligned}
& \left|e_{1}\right\rangle=\frac{1}{\sqrt{2}}\left(c_{+}^{\dagger} d_{-}^{\dagger}+c_{-}^{\dagger} d_{+}^{\dagger}\right)\left|\Psi_{\mathrm{sf}}^{M-1, M-1}\right\rangle, \\
& \left|e_{2}\right\rangle=\frac{1}{\sqrt{2}}\left(c_{+}^{\dagger} c_{-}^{\dagger}\left|\Psi_{\mathrm{sf}}^{M-2, M}\right\rangle+d_{-}^{\dagger} d_{+}^{\dagger}\left|\Psi_{\mathrm{sf}}^{M, M-2}\right\rangle\right)(\mathrm{A} 1)
\end{aligned}
$$

where \pm is used to denote the smallest quasi-momenta $\pm \delta k$. The inter- and intra-species interaction matrix elements in this excitation subspace are

$$
\left[\begin{array}{cc}
V\left(M+1-\frac{2}{M}\right)+U\left(M+\frac{1}{M}\right) & 2 U \sqrt{1-\frac{1}{M}} \\
2 U \sqrt{1-\frac{1}{M}} & V\left(M+1-\frac{3}{M}\right)+U M
\end{array}\right] .
$$

Upon diagonalizing this $2 \times 2$ matrix the resulting gap and non-degenerate excitations $\left|\Psi_{\mathrm{ex}, 1}^{M}\right\rangle$ and $\left|\Psi_{\mathrm{ex}, 2}^{M}\right\rangle$ (which will in general be some superposition of the states $\left|e_{1}\right\rangle$ and $\left.\left|e_{2}\right\rangle\right)$ are obtained in first-order perturbation theory.

In a similar way for subspace $\mathcal{S}_{M-2}$ the three lowestlying excitations are degenerate. This excitation subspace is again spanned by states where a single pair of atoms is expelled from the condensate to equal and opposite momenta with the lowest possible magnitude as

$$
\begin{aligned}
& \left|e_{1}\right\rangle=\frac{1}{\sqrt{2}}\left(c_{+}^{\dagger} c_{-}^{\dagger}\left|\Psi_{\mathrm{sf}}^{M-4, M+2}\right\rangle+d_{-}^{\dagger} d_{+}^{\dagger}\left|\Psi_{\mathrm{sf}}^{M+2, M-4}\right\rangle\right), \\
& \left|e_{2}\right\rangle=\frac{1}{2}\left(c_{+}^{\dagger} d_{-}^{\dagger}+c_{-}^{\dagger} d_{+}^{\dagger}\right)\left(\left|\Psi_{\mathrm{sf}}^{M+1, M-3}\right\rangle+\left|\Psi_{\mathrm{sf}}^{M-3, M+1}\right\rangle\right), \\
& \left|e_{3}\right\rangle=\frac{1}{\sqrt{2}}\left(c_{+}^{\dagger} c_{-}^{\dagger}\left|\Psi_{\mathrm{sf}}^{M, M-2}\right\rangle+d_{-}^{\dagger} d_{+}^{\dagger}\left|\Psi_{\mathrm{sf}}^{M-2, M}\right\rangle\right),
\end{aligned}
$$




$$
\left[\begin{array}{ccc}
V\left(M+1-\frac{4}{M}\right)+U\left(M-\frac{4}{M}\right) & U \sqrt{2\left(1-\frac{1}{M}-\frac{6}{M^{2}}\right)} & 0 \\
U \sqrt{2\left(1-\frac{1}{M}-\frac{6}{M^{2}}\right)} & V\left(M-1+\frac{2}{M}\right)+U\left(M-\frac{3}{M}\right) & U \sqrt{2\left(1-\frac{1}{M}-\frac{2}{M^{2}}\right)} \\
0 & U \sqrt{2\left(1-\frac{1}{M}-\frac{2}{M^{2}}\right)} & V\left(M+1+\frac{5}{M}\right)+U\left(M-\frac{4}{M}\right)
\end{array}\right]
$$

resulting in the non-degenerate excitations $\left|\Psi_{\mathrm{ex}, 1}^{M-2}\right\rangle$, $\left|\Psi_{\mathrm{ex}, 2}^{M-2}\right\rangle$ and $\left|\Psi_{\mathrm{ex}, 3}^{M-2}\right\rangle$.

\section{APPENDIX B: SF STATES}

In the $\mathrm{SF}$ regime $(U=V=0)$ the ground state in the subspace $\mathcal{S}_{2 n}$ is given by

$$
\left|e_{0}\right\rangle=\frac{1}{\sqrt{2}}\left(\left|\Psi_{\mathrm{sf}}^{2 n, 2 M-2 n}\right\rangle+\left|\Psi_{\mathrm{sf}}^{2 M-2 n, 2 n}\right\rangle\right)
$$

The excited states $\left|e_{l}\right\rangle$ are constructed by expelling pairs of atoms of opposite momentum $\pm k$. We can denote them by $\left|e_{l}\right\rangle \equiv\left|n_{0}, . ., n_{K}\right\rangle$ where $n_{k}=\frac{1}{2}\left\langle\mathrm{n}_{\mathrm{k}}^{\mathrm{a}}+\mathrm{n}_{\mathrm{k}}^{\mathrm{b}}\right\rangle$ and we omit the negative values because $n_{-k}=n_{k}$. An efficient way of generating the lowest eigenstates in ascending order in energy for large $M$ is to assume a quadratic approximation $E_{a p}(k) \sim k^{2}$ to the dispersion relation
$E(k)=2 J(1-\cos (2 \pi k / M))$ for the single particle energy in a tight-binding lattice. We can construct the eigenstates in ascending order of energy as

$$
\begin{aligned}
& n_{0}=N-\sum_{k=1}^{K} n_{k} \\
& n_{1}=m-\sum_{k=2}^{K} k^{2} n_{k} \\
& n_{k}=0, \ldots,\left\lfloor\frac{m-\sum_{q=2}^{k-1} q^{2} n_{q}}{k^{2}}\right\rfloor \quad k=2, . ., K
\end{aligned}
$$

where $m$ is an integer that runs from 0 to some maximum value $M_{m}$. Due to the color symmetry, the excited states $\left|e_{l}\right\rangle$ show a degeneracy $f(l)$ (see for example the first excited degenerate states Eq. (A1)) which can be calculated numerically.
[1] L. Pitaevskii and S. Stringari, Bose-Einstein Condensation (Oxford University Press, Oxford, 2003).

[2] M. Greiner et al., Nature (London) 415, 39 (2002); T. Stöferle et al., Phys. Rev. Lett. 92, 130403 (2004).

[3] D. Jaksch et al., Phys. Rev. Lett. 81, 3108 (1998); S.R. Clark and D. Jaksch, Phys. Rev. A 70, 043612 (2004).

[4] O. Mandel et al. , Nature 425, 937 (2003); D. Jaksch, H.-J. Briegel, J.I. Cirac, C.W. Gardiner and P. Zoller, Phys. Rev. Lett. 82, 1975 (1999).

[5] J. Dunningham, K. Burnett, R. Roth, W. D. Phillips, quant-ph/0608242 K. Mølmer and A. Sørensen, Phys. Rev. Lett. 82, 1835 (1999); A. Micheli, D. Jaksch, J. I. Cirac and P. Zoller, Phys. Rev. A 67, 013607 (2003).

[6] M. J. Holland and K. Burnett, Phys. Rev. Lett., 71, 1355 (1993); P. Bouyer and M. A. Kasevich, Phys. Rev. A 56, R1083 (1997).

[7] K. V. Kheruntsyan and P. D. Drummond, Phys. Rev. A 66, 031602 (2002).

[8] G.-B. Jo, Y. Shin, S. Will, T. A. Pasquini, M. Saba, W. Ketterle, D. E. Pritchard, M. Vengalattore, and M. Prentiss Phys. Rev. Lett. 98, 030407 (2007).

[9] M. Rodriguez, S. R. Clark and D. Jaksch, Phys. Rev. A 75, 011601 (R) (2007).

[10] A. Widera et al., Phys. Rev. Lett. 95, 190405 (2005); F. Gerbier, A. Widera, S. Fölling, O. Mandel, I. Bloch,
Phys. Rev. A 73, 041602(R) (2006) ; G. Thalhammer et al., Phys. Rev. Lett. 96, 050402(2006).

[11] J. Dziarmaga et al., Phys. Rev. Lett. 88, 167001 (2002).

[12] E. Altman, W. Hofstetter, E. Demler and M. D. Lukin, New J Phys 5, 113 (2003).

[13] T. Keilmann and J. J. García-Ripoll, condmat/0711.1483

[14] M. Rodriguez, S. R. Clark and D. Jaksch, J. Physics, Conf. Series XXX 2007.

[15] S. Fölling, F. Gerbier, A. Widera, O. Mandel, T. Gericke and I. Bloch, Nature 434, 481 (2005); E. Altman, E. Demler and M. D. Lukin, Phys. Rev. A 70, 013603 (2004).

[16] E. A. Donley, N. R. Claussen, S. T. Thompson, and C. E. Wieman, Nature 417, 529 (2002); A. Marte et al., Phys. Rev. Lett. 89, 283202 (2002); M. Theis et al., Phys. Rev. Lett. 93, 123001 (2004).

[17] L. Amico, A. Osterloh and F. Cataliotti, Phys. Rev. Lett. 95, 063201 (2005).

[18] Z. Nussinov and G. Ortiz, cond-mat/0702377

[19] L. D. Landau and E. M. Lifshitz, Quantum Mechanics: Non-relativistic Theory (Butterworth-Heinemann, 2005) Vol 3, $\S 79$ and $\S 96$.

[20] J. von Neumann and E. Wigner, Z. Phys. 30, 467 (1929).

[21] L. Landau, Sov. Phys. 1, 89 (1932); Z. Phys. Sov. 2, 
1932(1932); C. Zener, Proc. R. Soc. London, Ser. A 137, 696 (1932).

[22] S. Brundobler and V. Elser, J. Phys. A 261211 (1993).

[23] A. R. Kolovsky and A. Buchleitner, Europhys. Lett., 68, 632 (2004).

[24] L. C. Biedenharn and J. D. Louck, Angular Momentum in Quantum Physics. Theory and application. Reading, MA, Addison-Wesley 1981 (pp.49).

[25] B. Yurke, S. L. McCall and J. R. Klauder, Phys. Rev. A 33, 4033 (1986).

[26] V. Giovanetti, S. Lloyd and L. Maccone, Science 306, 1330 (2004) and references therein.

[27] J.J. Bollinger, W.M. Itano, D.J. Wineland and D.J. Heinzen, Phys. Rev. A 54, R4649 (1996); S. F. Huelga, et al Phys. Rev. Lett. 79, 3865 (1997).

[28] T. Kim, Y. Ha, J. Shin, H. Kim, G. Park, K. Kim, T.G. Noh and C. K. Hong, Phys. Rev. A 60, 708 (1999).

[29] J.A. Dunningham and K. Burnett, Phys. Rev. A 70,
033601 (2004); T. Kim et al., ibid. 60, 708 (1999).

[30] B. Oles and K. Sacha, cond-mat/0701.212

[31] The sequence is $1,2,3,7,13,35,85,257,765,2518,8359$, 28968, 101340, 361270, 1297879, 4707969, 17179435, 63068876, 232615771, 861725794.. and can be found in http://www.research.att.com/ njas/sequences/A006840

[32] http://www.research.att.com/ njas/sequences/A073020 http://www.research.att.com/ njas/sequences/A078925 The full series and the generating algorithm is given in http://www.research.att.com/ njas/sequences/A052307

[33] Except for even $M$ where $\left|\Psi_{\mathrm{ps}}^{N / 2}\right\rangle=\left|\psi_{\mathrm{ps}}^{N / 2}\right\rangle$.

[34] Except for $M=N / 2$ even and $n=N / 4$ where the degeneracy his given by $\left(\begin{array}{l}N / 2 \\ N / 4\end{array}\right)$.

[35] It is clear, for example, that quantum melting of the $\mathrm{BHM}$ is not a single-particle process since no product of single-particle unitaries can connect a MI state with a non-interacting SF. 